\title{
SIRT3 protects from hypoxia and staurosporine- mediated cell death by maintaining mitochondrial membrane potential and intracellular $\mathrm{pH}$
}

\author{
L Pellegrini ${ }^{1}$, B Pucci ${ }^{2}$, L Villanova ${ }^{1}$, ML Marino ${ }^{3}$, G Marfe ${ }^{4}$, L Sansone ${ }^{1}$, E Vernucci $^{1}$, D Bellizzi ${ }^{5}$, V Reali ${ }^{1}$, M Fini $^{2}$, MA Russo ${ }^{1,2}$ \\ and M Tafani ${ }^{* 1}$
}

Mitochondrial sirtuin 3 (SIRT3) mediates cellular resistance toward various forms of stress. Here, we show that in mammalian cells subjected to hypoxia and staurosporine treatment SIRT3 prevents loss of mitochondrial membrane potential $\left(\Delta \Psi_{\mathrm{mt}}\right)$, intracellular acidification and reactive oxygen species accumulation. Our results indicate that: (i) SIRT3 inhibits mitochondrial permeability transition and loss of membrane potential by preventing HKII binding to the mitochondria, (ii) SIRT3 increases catalytic activity of the mitochondrial carbonic anhydrase VB, thereby preventing intracellular acidification, Bax activation and apoptotic cell death. In conclusion we propose that, in mammalian cells, SIRT3 has a central role in connecting changes in $\Delta \Psi_{\mathrm{mt}}$, intracellular $\mathrm{pH}$ and mitochondrial-regulated apoptotic pathways.

Cell Death and Differentiation (2012) 19, 1815-1825; doi:10.1038/cdd.2012.62; published online 18 May 2012

Sirtuins are a family of protein highly conserved in both prokaryotes and eukaryotes. ${ }^{1}$ The name derives from the Saccharomyces cerevisiae gene silent information regulator 2 (Sir2), the first known sirtuin, that, by silencing chromatin via deacetylation of histones, regulates both replicative and overall life span. ${ }^{2}$ In mammals, seven Sir2 homologs, designated SIRT1 through SIRT7 have been identified, that regulate a wide range of intracellular processes including metabolism, longevity, aging, cancer and response to stress. $^{3-5}$ These proteins are characterized by an evolutionarily conserved sirtuin core domain ${ }^{6}$ that contains the catalytic and nicotinamide adenine dinucleotide $\left(\mathrm{NAD}^{+}\right)$binding domain. Each sirtuin catalyzes protein deacetylation or adenosine diphosphate (ADP) ribosylation in vitro, requires $\mathrm{NAD}^{+}$for enzymatic activity and generates nicotinamide, which then acts as a negative feedback inhibitor. ${ }^{7}$ Because of the $\mathrm{NAD}^{+}$requirement, sirtuins are categorized as class III histone deacetylases. ${ }^{8}$

Mammalian sirtuins show distinct acetylated protein substrates and are localized in distinct subcellular compartments. Sirtuin 1 (SIRT1), SIRT6 and SIRT7 are in the nucleus, SIRT2 is primarily cytosolic and SIRT3, SIRT4 and SIRT5 are in the mitochondria. ${ }^{9}$ Mitochondrial sirtuins participate in the regulation of ATP production, metabolism, apoptosis and cell signaling. ${ }^{10}$ Among the three mitochondrial sirtuins, SIRT3 controls the global lysine acetylation of these organelles. ${ }^{11}$
SIRT3 mediates cellular resistance toward various forms of stress by maintaining genomic stability and mitochondrial integrity. For example, SIRT3 influences cell survival by regulating the state of the mitochondrial permeability transition (MPT). However, the precise effects of SIRT3 on MPT are still not clear. In fact, some reports have shown that SIRT3 would deacetylate and inhibit the enzymatic activity of cyclophilin $D^{12}$ with the following dissociation from adenine nucleotide translocator, which, in turn, promotes detachment of hexokinase II (HKII) from voltage-dependent anion channel. Inhibition of cyclophilin D is thought to decrease the probability of pore opening. ${ }^{13}$ Importantly, inhibition of SIRT3 activity by treating cells with ethanol enhanced the sensitivity of mitochondria to induction of MPT by increasing cyclophilin D activity. ${ }^{14}$ By contrast, it has also been reported that in some instances cyclophilin $\mathrm{D}$ prevents apoptotic cell death through modulation of $\mathrm{Bcl}-2$ and by promoting the binding of HKII to the mitochondria. ${ }^{15,16}$ Shulga et al. ${ }^{12}$ demonstrated that a decrease of SIRT3 activity enhances the binding of HKII to the mitochondria by increasing cyclophilin D activity, potentially making the cells resistant to apoptosis.

Apoptotic cell death is characterized by well-defined biochemical and morphological changes such as plasma membrane blebbing, DNA fragmentation and changes in the MPT with release of mitochondrial proteins. ${ }^{17}$ Mitochondria have a central role in regulating the apoptotic process because many apoptogenic proteins such as cytochrome $c$,

\footnotetext{
${ }^{1}$ Department of Experimental Medicine, Sapienza University of Rome, Rome, Italy; ${ }^{2}$ Department of Cellular and Molecular Pathology, IRCCS San Raffaele Pisana, Rome, Italy; ${ }^{3}$ Department of Therapeutic Research and Medicines Evaluation, Unit of Antitumor Drugs, Istituto Superiore di Sanità, Rome, Italy; ${ }^{4}$ Department of Experimental Medicine and Biochemical Sciences, University of Rome 'Tor Vergata', Rome, Italy and ${ }^{5}$ Department of Cell Biology, University of Calabria, Rende, Italy ${ }^{*}$ Corresponding author: M Tafani, Department of Experimental Medicine, Sapienza University of Rome, Viale Regina Elena 324, Rome, Italy. Tel +39-6-49970665; Fax: +39-6-49970806; E-mail: marcotafani@yahoo.com Keywords: SIRT3; mitochondria; ROS; intracellular pH

Abbreviations: Sir2, silent information regulator 2; MPT, mitochondrial permeability transition; OMM, outer mitochondrial membrane; PTP, permeability transition pore; AIF, apoptosis-inducing factor; CSA, cyclosporin A; PARP, poly(ADP-ribose)polymerase; PI, propidium iodide; LDH, lactate dehydrogenase; HKII, hexokinase II; $\mathrm{CA}$, carbonic anhydrase; HMGB1, high mobility group box 1; NAD, nicotinamide adenine dinucleotide; NAC, N-acetyl-cysteine; ROS, reactive oxygen species; Phb, prohibitin

Received 26.7.11; revised 02.4.12; accepted 07.4.12; Edited by E Gottlieb; published online 18.5.12
} 
apoptosis-inducing factor (AIF), caspases and so on, normally present in these organelles, are released following apoptotic damage and amplify the apoptotic process. ${ }^{18}$ In particular, mitochondrial damage is accompanied by induction of the MPT and loss of mitochondrial membrane potential $\left(\Delta \Psi_{\mathrm{mt}}\right)$, events that have proapoptotic proteins as main effectors. In fact, insertion of proapoptotic proteins such as Bax, Bid, tBid, Bad and so on into the mitochondrial membrane and/or their association to the pore components, causes the release of cytochrome $c$ and activation of downstream effectors caspases. ${ }^{19}$ The proapoptotic protein Bax is a potent proapoptotic protein capable of inducing all the hallmarks of apoptosis. ${ }^{20}$ Bax activation is a prerequisite for its apoptotic function. One model of Bax activation proposes that a change in $\mathrm{pH}$ of the cytosol alters the conformation of the protein, an effect that results in exposure of the membrane-targeting C-terminal domain and translocation to the mitochondria. ${ }^{21}$ SIRT3 has been shown to be involved in preventing apoptotic cell death in different models; however, its role in controlling intracellular $\mathrm{pH}\left(\mathrm{pH}_{\mathrm{i}}\right)$ has never been addressed before. Similarly, association between changes in $\Delta \Psi_{\mathrm{mt}}$ and SIRT3 expression levels are still not well defined.

The present study investigated the mechanisms through which SIRT3 protects different cell lines from hypoxia or staurosporine (STS)-mediated cell death. SIRT3-increased expression inhibited MPT induction, loss of membrane potential and reactive oxygen species (ROS) accumulation. Moreover, SIRT3 prevented HKII binding to the mitochondria and increased CA VB activity, thereby maintaining a physiological $\mathrm{pH}_{\mathrm{i}}$. Finally, by preventing intracellular acidification, SIRT3 inhibits mitochondrial translocation of Bax and maintains mitochondrial membrane integrity.

\section{Results}

SIRT3 protects cells from both apoptotic and necrotic cell death. The protective role of SIRT3 in MDA-MB-231 and HeLa cells was evaluated by stably silencing or overexpressing this sirtuin (Supplementary Figure S1). SIRT3 activity in mitochondrial fractions of MDA-MB-231 and HeLa cells was increased in overexpressing and decreased in silenced cells compared with control untrasfected cells (Supplementary Figure S1). Figure 1a shows percentage of cell death measured by propidium iodide (PI)/ Annexin V staining in WT, SIRT3-overexpressing or silenced cells treated with hypoxia or STS $250 \mathrm{nM}$ for $48 \mathrm{~h}$. Hypoxia was used to induce necrotic cell death, whereas STS was used to induce apoptosis. It is worth noting, however, that when treating cells with hypoxia or STS, we never observed a clear $\mathrm{Pl}^{+}$population but always $\mathrm{PI}^{+} /$Annexin $\mathrm{V}^{+}$cells. This is probably due, as also reported by Krisko et al., ${ }^{17}$ to the externalization by necrotic cells of phosphatidylserine during hypoxia. After $48 \mathrm{~h}$ of hypoxia, $11 \%$ of WT cells were $\mathrm{Pl}^{+} /$Annexin $\mathrm{V}^{+}$, whereas $8 \%$ were $\mathrm{PI}^{-} /$Annexin $\mathrm{V}^{+}$ (Figure 1a). Overexpression of SIRT3 reduced $\mathrm{PI}^{+} /$Annexin $\mathrm{V}^{+}$cells to $2 \%$ and $\mathrm{PI}^{-} /$Annexin $\mathrm{V}^{+}$to $5 \%$. SIRT3 silencing increased $\mathrm{PI}^{+} /$Annexin $\mathrm{V}^{+}$to $15 \%$ and $\mathrm{PI}^{-} /$Annexin $\mathrm{V}^{+}$cells to $13 \%$. In the presence of STS, $\mathrm{PI}^{+} /$Annexin $\mathrm{V}^{+}$cells were 12, 4 and $20 \%$, whereas
$\mathrm{PI}^{-} /$Annexin $\mathrm{V}^{+}$cells were $30 \%, 8 \%$ and $52 \%$ for $\mathrm{WT}$, SIRT3-overexpressing and SIRT3-silenced cells, respectively (Figure 1a). Images and percentage of cell killing after hypoxia and STS treatment are shown in Figure 1b. Moreover, images and total number of apoptotic nuclei counted after Hoechst staining are also reported in Figure 1c. Importantly, cyclosporin A (CsA), an inhibitor of the MPT pore ${ }^{22}$ reduced SIRT3-silenced hypoxic cell death to a percentage similar to that of WT cells (Figure 1b). Similar results were obtained in WT, SIRT3-overexpressing and SIRT3-silenced HeLa cells exposed to hypoxia (Supplementary Figure S2A). Interestingly, SIRT3 protection was observed also after 7 days of hypoxia when only $30 \%$ of SIRT3 overexpressing cells were killed (Supplementary Figure S2B). By contrast, at the same time, $65 \%$ of WT cells and $90 \%$ of SIRT3-silenced cells were dead (Supplementary Figure S2B).

In our model, we assessed, in the extracellular medium, lactate dehydrogenase (LDH) as an accurate measure of cell membrane integrity and cell death, and high mobility group box 1 (HMGB1) protein release as an accurate measure of necrotic cell death. ${ }^{17}$ In fact, HMGB1 is a DNA-associated protein that remains bound to DNA during apoptosis, being released only by necrotic cells. ${ }^{17}$ Figure $2 \mathrm{a}$ shows that $72 \mathrm{~h}$ of hypoxia significantly increased LDH release in WT cells. Overexpression of SIRT3 reduced LDH release, whereas SIRT3 silencing caused a burst in LDH released into the culture medium (Figure $2 a$ ). Figure $2 b$ shows that WT cells released HMGB1 after $72 \mathrm{~h}$ of hypoxia. By 6 days of hypoxia, there was a clear accumulation of HMGB1. SIRT3-overexpressing cells showed a reduced accumulation of HMGB1 in the extracellular medium both at $72 \mathrm{~h}$ and 6 days (Figure 2b). By contrast, SIRT3-silenced cells showed HMGB1 release under normoxia that was significantly increased after $72 \mathrm{~h}$ and 6 days of hypoxia (Figure $2 \mathrm{~b}$ ).

SIRT3 effects on $\Delta \Psi_{\mathrm{mt}}$ and $\mathrm{pH}_{\mathrm{i}}$. MPT induction with loss of $\Delta \Psi_{\mathrm{mt}}$ is considered a key event in the control of apoptotic and necrotic cell death. ${ }^{18}$ The effects of SIRT3 on $\Delta \Psi_{\mathrm{mt}}$ was evaluated in WT, SIRT3-overexpressing and SIRT3-silenced cells. Figure 3 a shows that under normoxia $8 \%$ of WT cells had a low $\Delta \Psi_{\mathrm{mt}}$. By contrast, about $25 \%$ of SIRT3-silenced cells had a low $\Delta \Psi_{\mathrm{mt}}$ under the same conditions (Figure 3a). Interestingly, only $4 \%$ of SIRT3-overexpressing cells showed a low $\Delta \Psi_{\mathrm{mt}}$ (Figure 3a). The number of cells with reduced $\Delta \Psi_{\mathrm{mt}}$ increased after $24 \mathrm{~h}$ of hypoxia. In fact, Figure $3 \mathrm{a}$ also shows that $20 \%$ of WT cells had a low $\Delta \Psi_{\mathrm{mt}}$ when under hypoxia. Such a percentage was 10\% in SIRT3-overexpressing cells and $>30 \%$ in SIRT3-silenced cells (Figure $3 a$ ). Similarly, STS caused $\Delta \Psi_{\mathrm{mt}}$ loss in $25 \%$ of WT, $12 \%$ of SIRT3-overexpressing and $27 \%$ of SIRT3-silenced cells (Figure 3a). Mitochondria are the main source of ROS and SIRT3 has been shown to modulate the activity of crucial enzymes involved in ROS scavenging. ${ }^{23}$ Therefore, ROS production was measured in WT and SIRT3 clones. Supplementary figure 3 shows that $48 \mathrm{~h}$ of hypoxia treatment increased intracellular ROS in WT cells, an effect reduced by the ROS scavenger $\mathrm{N}$-acetyl-cysteine (NAC). Overexpression of SIRT3 reduced both basal and hypoxia-induced accumulation of ROS (Supplementary Figure S3). By contrast, SIRT3 silencing significantly increased ROS 

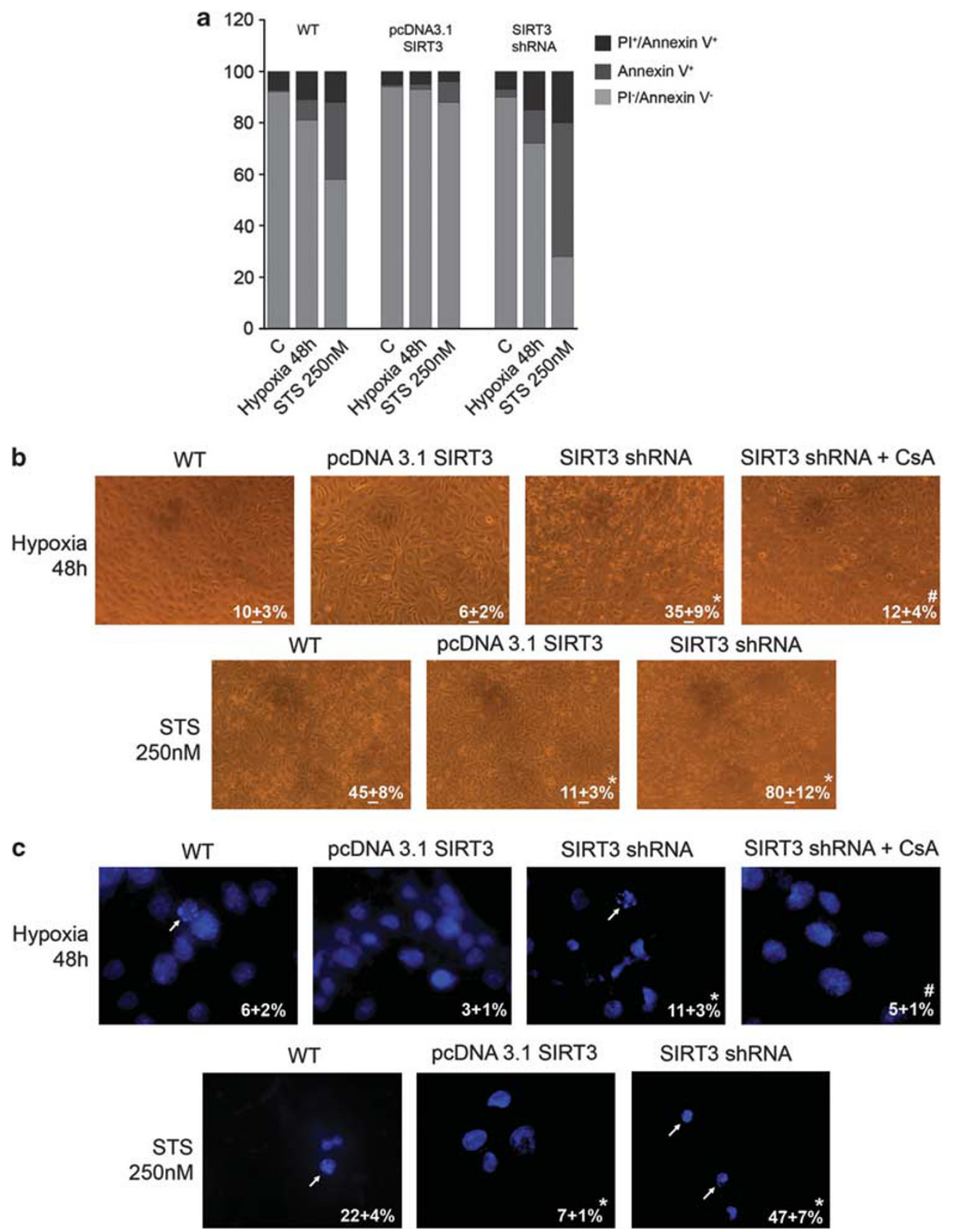

Figure 1 SIRT3 prevents cell death after hypoxia or STS treatment. (a) MDA-MB-231 WT, SIRT3-overexpressing and SIRT3-silenced cells were incubated under normoxia or hypoxia for $48 \mathrm{~h}$. Alternatively, MDA-MB-231 WT, SIRT3-overexpressing and SIRT3-silenced cells were treated with STS $250 \mathrm{nM}$ for $48 \mathrm{~h}$. Following treatment,

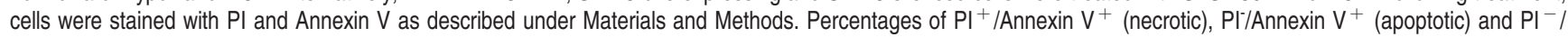
Annexin $\mathrm{V}^{-}$(vital) cells are reported as bar graphs. (b) MDA-MB-231 WT, SIRT3-overexpressing and SIRT3-silenced cells were incubated under normoxia or hypoxia for $48 \mathrm{~h}$. Furthermore, SIRT3-silenced cells were also incubated under hypoxia in the presence of CsA $5 \mu \mathrm{m}$. Alternatively, MDA-MB-231 WT, SIRT3-overexpressing and SIRT3silenced cells were treated with STS $250 \mathrm{nM}$ for $48 \mathrm{~h}$. Pictures of the cells were taken at $\times 20$ with a digital camera mounted on an EclipseNet 2000 microscope. Percentage of cell death was determined by Trypan blue exclusion. Values are represented as mean \pm S.D. *Significantly different from WT. "Significantly different from SIRT3-silenced cells. Significance was set at $P<0.05$. (c) MDA-MB-231 WT, SIRT3-overexpressing and SIRT3-silenced cells were incubated under normoxia or hypoxia for $48 \mathrm{~h}$. Furthermore, SIRT3-silenced cells were also incubated under hypoxia in the presence of CsA $5 \mu \mathrm{M}$. Alternatively, MDA-MB-231 WT, SIRT3-overexpressing and SIRT3silenced cells were treated with STS $250 \mathrm{nM}$ for $48 \mathrm{~h}$. Following treatment cells were stained with Hoechst as described under 'Materials and Methods'. Pictures of apoptotic nuclei were taken at $\times 60$ with an AxioCam digital camera mounted on an Zeiss LSM 510 fluorescent microscope. Apoptotic and intact nuclei from five different fields for a total of 150 cells were counted and values reported in each image. Values are represented as mean \pm S.D. *Significantly different from WT. "Significantly different from SIRT3silenced cells. Significance was set at $P<0.05$. Apoptotic nuclei are indicated by white arrows

accumulation with only a partial reduction by NAC (Supplementary Figure S3). Interestingly, NAC also reduced SIRT3 expression in WT cells under both normoxic and hypoxic conditions (Supplementary Figure S3 right side).
Maintenance of $\mathrm{pH}_{\mathrm{i}}$ within the physiological range is important because of its effect on a number of enzymes, on the efficiency of the contractile elements, on ion channels and on the cell cycle. ${ }^{24}$ Alteration of $\mathrm{pH}_{\mathrm{i}}$ affects metabolic enzymes 
a

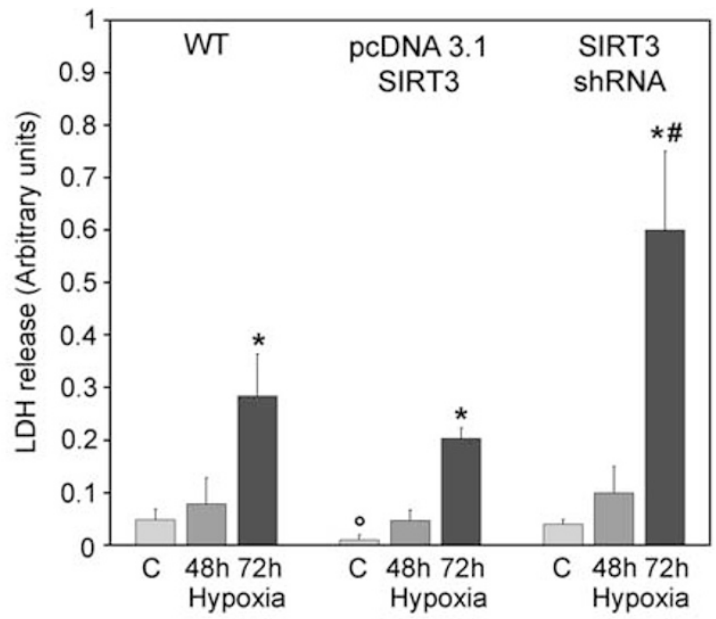

b

\begin{tabular}{|c|c|c|c|c|c|c|c|}
\hline \multicolumn{2}{|l|}{ WT } & \multicolumn{3}{|c|}{ pcDNA 3.1 SIRT3 } & \multicolumn{3}{|c|}{ SIRT3 shRNA } \\
\hline Hyp & $x i a$ & & Hyp & & & & \\
\hline $72 \mathrm{~h}$ & $6 d$ & C & $72 \mathrm{~h}$ & $6 d$ & C & $72 \mathrm{~h}$ & $6 d$ \\
\hline
\end{tabular}

Figure 2 SIRT3 reduces LDH and HMGB1 release. (a) MDA-MB-231 WT, SIRT3-overexpressing and SIRT3-silenced cells were incubated under normoxia, hypoxia for 48 and $72 \mathrm{~h}$. Activity of LDH released by the cells was measured as described under Materials and Methods. *Significantly different from control normoxic and hypoxia $48 \mathrm{~h}$. ${ }^{\circ}$ Significantly different from control WT and SIRT3silenced cells. "Significantly different from $72 \mathrm{~h}$ hypoxia of WT and SIRT3overexpressing cells. Significance was set at $P<0.05$. (b) MDA-MB-231 WT, SIRT3-overexpressing and SIRT3-silenced cells were incubated under normoxia, hypoxia for $72 \mathrm{~h}$ and 6 days. Afterward, cellular medium was collected. HMGB1 extracellular levels were determined by western Blot. C, control normoxic cells

activity, protein synthesis as well as many other cellular processes. ${ }^{24}$ We observed that acidification of the growth medium was accelerated in SIRT3-silenced cells compared with WT or SIRT3-overexpressing cells. For this reason we proceeded by measuring both intracellular and extracellular $\mathrm{pH}$. Figure $3 \mathrm{~b}$ shows that SIRT3 silencing caused a significant drop in $\mathrm{pH}_{\mathrm{i}}$ that was more evident under hypoxia. WT cells showed a decrease in $\mathrm{pH}_{\mathrm{i}}$ after $48 \mathrm{~h}$ of hypoxia treatment. By contrast, no intracellular acidification was observed in SIRT3overexpressing cells (Figure $3 b$ ).

Finally, increased proton production and extrusion was measured by determining culture medium acidification following 24 and $48 \mathrm{~h}$ of hypoxia. Figure $3 \mathrm{c}$ shows that hypoxia increased extracellular acidification in SIRT3-silenced cells compared with WT cells. By contrast, SIRT3 overexpression prevented extracellular acidification (Figure $3 c$ ).

SIRT3 effects on HKII mitochondrial binding and carbonic anhydrase (CA) activity. In order to unravel the mechanisms involved in the alterations of intracellular and extracellular $\mathrm{pH}$ that we observed following SIRT3 overexpression or silencing, we focused our attention on HKII and CA enzymes. Our choice is motivated by the fact that SIRT3 has been shown to modulate HKII binding to the outer mitochondrial membrane (OMM), thereby regulating both the opening/closing of the permeability transition pore (PTP) and the glycolytic rate. $^{12,22}$ Figure $4 a$ shows that hypoxia increased HKII expression. In fact, the decrease observed after $72 \mathrm{~h}$ of hypoxia in SIRT3-silenced cells is probably due to the increasing cell death rate. Next, we measured HKII content in the mitochondrial and cytosolic fractions from WT and SIRT3 clones (Figure 4a). In WT cells, HKII accumulated in the mitochondrial fraction. However, in SIRT3-overexpressing cells such an accumulation was absent during the first $36 \mathrm{~h}$ of hypoxia when HKII was mostly cytosolic. After $72 \mathrm{~h}$ of hypoxia we observed some HKII mitochondrial translocation (Figure 4a). By contrast, SIRT3-silenced cells had a high HKII content in the mitochondria under normoxia that did not change after $36 \mathrm{~h}$ of hypoxia (Figure 4a). Increase in glycolytic rate was measured through the lactate released in the cellular medium. Figure $4 \mathrm{~b}$ shows that both normoxic and hypoxic SIRT3-overexpressing cells released less lactate compared with WT cells. By contrast, SIRT3-silenced cells had an increased accumulation of lactate in the extracellular medium both under normoxia and hypoxia (Figure 4b).

To our knowledge, no data have been published on SIRT3 and CA. However, CA is an enzyme that has been shown to have a major role in the control of $\mathrm{pH}_{\mathrm{i}}{ }^{25} \mathrm{CA}$ catalyzes the reversible reaction: $\mathrm{CO}_{2}+\mathrm{H}_{2} \mathrm{O} \leftrightarrow \mathrm{H}^{+}+\mathrm{HCO}_{3}^{-}$. In mammals, $16 \mathrm{CA}$ isoforms have been discovered so far. Among these, the hypoxia-induced CA IX has a plasma membrane localization, whereas the less-studied CA VB localizes to the mitochondrial matrix where it controls $\mathrm{CO}_{2} / \mathrm{HCO}_{3}^{-}$balance and Krebs cycle stimulation. ${ }^{25}$ Hypoxia incubation of WT and SIRT3-overexpressing cells significantly increased CA IX expression and activity (Figures $4 \mathrm{C}$ and d). In SIRT3-silenced cells there was a significant increase of CA IX in normoxic cells, that was also accompanied by an increased catalytic activity (Figures $4 \mathrm{C}$ and d). Again, hypoxia stimulated CA IX synthesis and activity (Figures $4 \mathrm{c}$ and d). SIRT3 and CA VB are both localized in the mitochondrial matrix; therefore, we examined CA VB expression. Figure 4e shows that hypoxia did not significantly increase CA VB levels. However, in WT cells, hypoxia increased CA VB catalytic activity (Figure 4f). Interestingly, SIRT3 overexpression significantly increased CA VB catalytic activity both under normoxia and hypoxia compared with WT and silenced cells. By contrast, in SIRT3silenced cells, CA VB catalytic activity was significantly reduced (Figure 4f).

SIRT3 effects on mitochondrial apoptotic pathway. SIRT3 controls HKII binding to the PTP that in turn promotes release of proapoptotic factors. ${ }^{12}$ On the other hand, SIRT3 controls CA VB activity and consequently $\mathrm{pHi}$. Intracellular acidification represents a mechanism that causes conformational change and mitochondrial accumulation of the proapoptotic protein Bax. ${ }^{21}$ Mitochondrial Bax exacerbates PTP opening with release of apoptogenic factors. ${ }^{21}$ Mitochondrial accumulation of Bax in the presence of hypoxia or STS is shown in Figure 5a. In WT and SIRT3-silenced cells, $36 \mathrm{~h}$ hypoxia exposure resulted in Bax translocation from cytosol to mitochondria. By contrast, SIRT3-overexpressing cells did not show any mitochondrial accumulation of Bax (Figure 5a). Again, STS treatment increased Bax in the mitochondrial fraction of both WT and SIRT3-silenced cells, whereas Bax accumulation was absent in the mitochondria of 

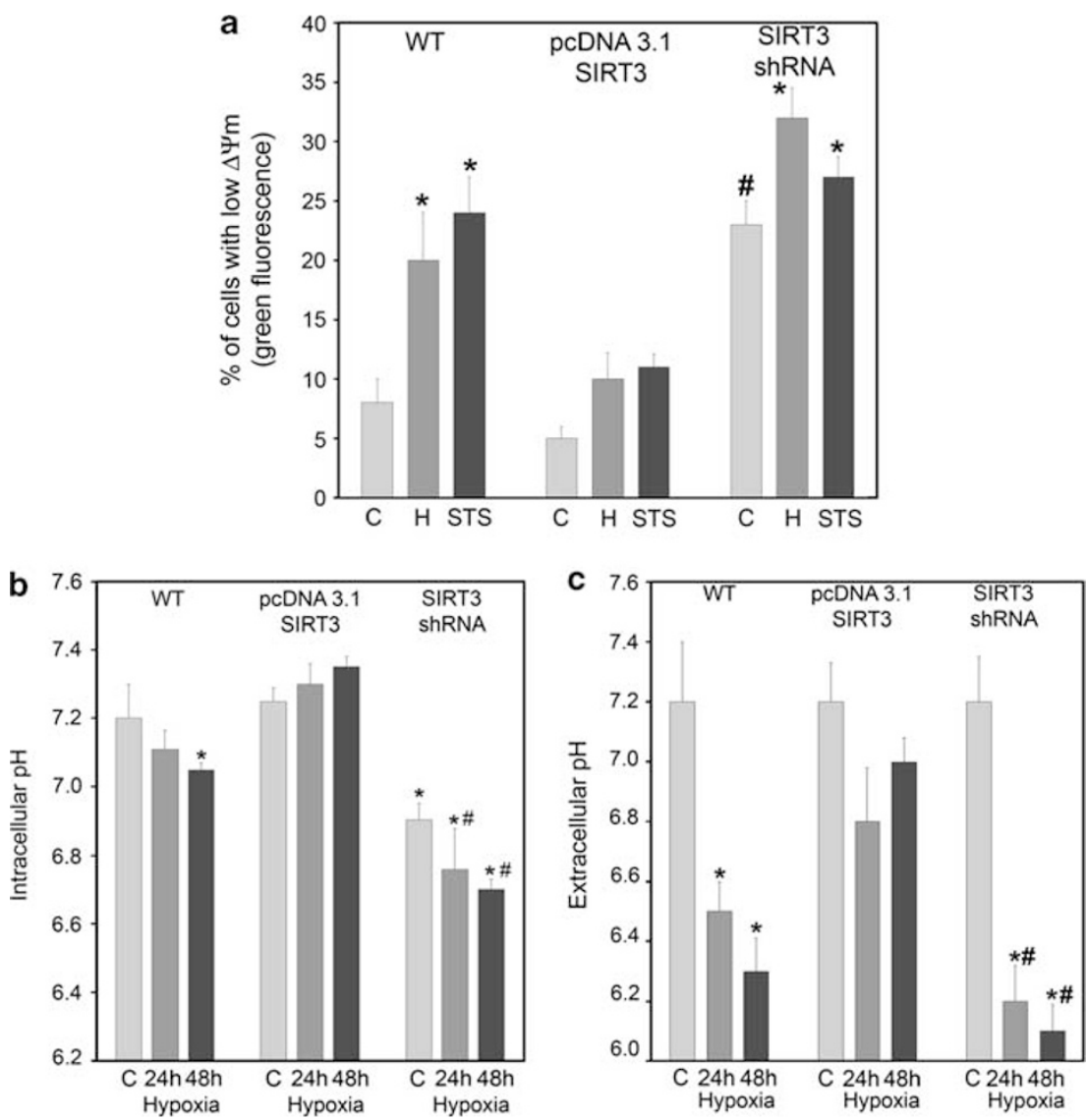

Figure 3 SIRT3 prevents MPT induction and intracellular/extracellular acidification. (a) MDA-MB-231 WT, SIRT3-overexpressing and SIRT3-silenced cells were incubated under normoxia, hypoxia or STS for $24 \mathrm{~h}$. Changes in $\Delta \Psi_{\mathrm{mt}}$ were determined by using the $\Delta \Psi_{\mathrm{mt}}$ sensitive dye JC-1. Fluorescence intensity was measured by flow cytometry as described under Materials and Methods. Data are representative of at least three separate experiments. Values are represented as mean \pm S.D. *Significantly different from control normoxic cells. "Significantly different from control normoxic cells of WT and SIRT3-overexpressing clones. Significance was set at $P<0.05$. (b) MDA-MB-231 WT, SIRT3-overexpressing and SIRT3-silenced cells were incubated under normoxia or hypoxia for 24 and $48 \mathrm{~h}$. $\mathrm{pH}_{\mathrm{i}}$ was evaluated by flow cytometry using the pH-sensitive fluorescent probe BCECF-AM as described under Materials and Methods. Data are representative of at least three separate experiments. *Significantly different from control normoxic WT and SIRT3-overexpressing cells. "Significantly different from control SIRT3-silenced cells and from hypoxia-treated WT and SIRT3-overexpressing cells. Significance was set at $P<0.05$. (c) MDA-MB-231 WT, SIRT3-overexpressing and SIRT3-silenced cells were incubated under normoxia or hypoxia for 24 and $48 \mathrm{~h}$. Extracellular pH in culture medium was determined as described under Materials and Methods. *Significantly different from control normoxic cells. "Significantly different from hypoxia of WT and SIRT3-overexpressing cells. Significance was set at $P<0.05$. C, control normoxic cells

SIRT3-overexpressing cells (Figure 5a right side). Moreover, whole cellular levels of the antiapoptotic protein Bcl-2 were significantly increased only in SIRT3-overexpressing cells (Figure 5b).

Induction of MPT, followed by cytochrome $c$ release from mitochondria, is an important step of the apoptotic process. ${ }^{19}$ Figure $5 \mathrm{c}$ shows that in WT MDA-MB-231 cells hypoxia caused cytochrome $c$ release from mitochondria and accumulation in the cytosol at $72 \mathrm{~h}$. SIRT3 overexpression inhibited cytochrome $c$ release, whereas SIRT3 silencing induced a significant loss of cytochrome $c$ from the mitochondria (Figure $5 \mathrm{c}$ ). Progression of the apoptotic process was documented by the cleavage of the caspase 3 substrate poly(ADP-ribose) polymerase (PARP). After $72 \mathrm{~h}$ of hypoxia, PARP was cleaved in WT and SIRT3-silenced cells, whereas no cleavage was observed in SIRT3-overexpressing cells (Figure 5c). Similarly, STS treatment was followed by cytochrome $c$ release and PARP cleavage in WT and in SIRT3-silenced cells. No cytochrome $c$ release and PARP cleavage was observed in SIRT3-overexpressing cells (Figure $5 \mathrm{c}$ right side).

Another documented effect of mitochondrial damage and apoptosis is the release of the AIF that accumulates in the nucleus causing DNA degradation. ${ }^{26}$ Figure $5 \mathrm{~d}$ shows AIF nuclear accumulation in WT and SIRT3-silenced cells following hypoxia or STS treatment. By contrast, SIRT3 overexpression completely inhibited nuclear accumulation of AlF (Figure 5d).

Hypoxia increases SIRT3 expression via SP1. As SIRT3 levels influences cellular metabolism and hypoxia represents a metabolic stress, we investigated changes in SIRT3 levels following hypoxia exposure. In fact, those changes may represent an adaptive cellular response to hypoxia that contributes to cell survival under such a stress. Figure $6 a$ shows that hypoxic incubation of MDA-MB-231 cells increased mitochondrial expression and activity of SIRT3 after 17 and $24 \mathrm{~h}$. Hypoxia regulation of SIRT3 expression 
was confirmed also in HeLa and K562 cell lines. Supplementary figure 4 shows that in K562 cells SIRT3 expression and activity increased after $17 \mathrm{~h}$ to decrease after 48 h (Supplementary Figure S4A). In HeLa cells, SIRT3 expression and activity increased from 17 to $72 \mathrm{~h}$ (Supplementary Figure S4B).

In order to evaluate whether SIRT3 increase under hypoxia was mediated by HIF- $1 \alpha$, HIF- $1 \alpha$-silenced cell lines were produced (Figure 6b). Figure 6b (right side) shows that SIRT3 levels were still increased, in HIF- $1 \alpha$-silenced cells, after 4 and $6 \mathrm{~h}$ of hypoxia.

SP1 binding sites are clustered in proximity of the SIRT3 transcription start site. ${ }^{27}$ Therefore, stable cell lines silenced for SP1 were produced (Figure 6c). Figure 6c (right side) shows that in SP1-silenced cells SIRT3 expression was not detectable under normoxia and barely detectable under a

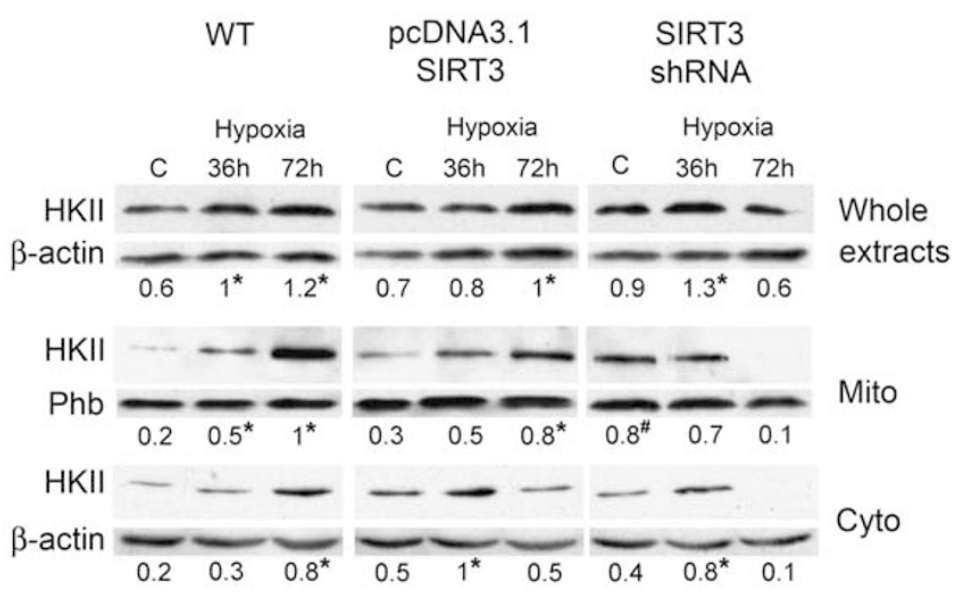

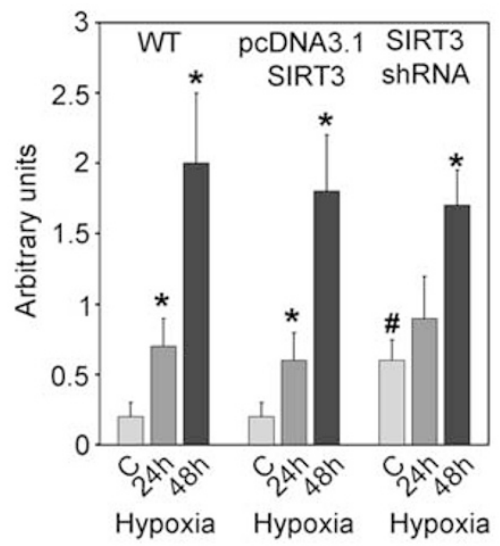
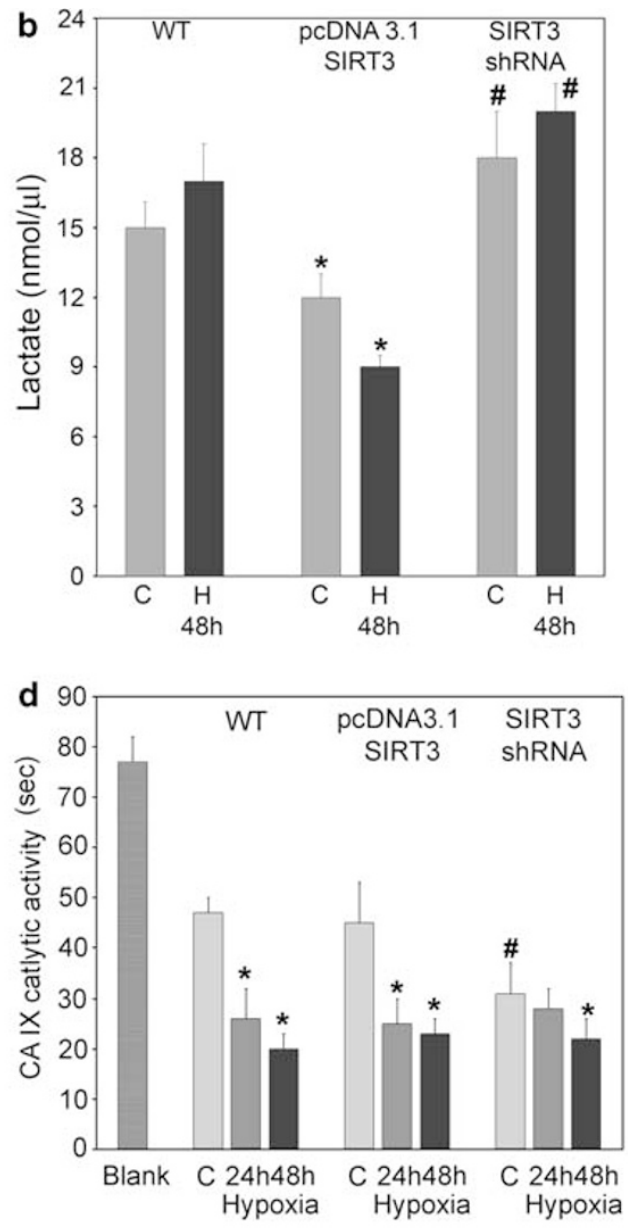

e

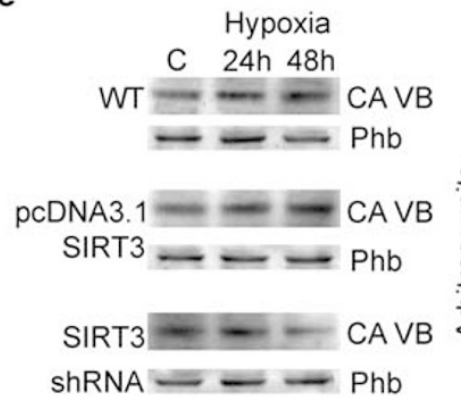

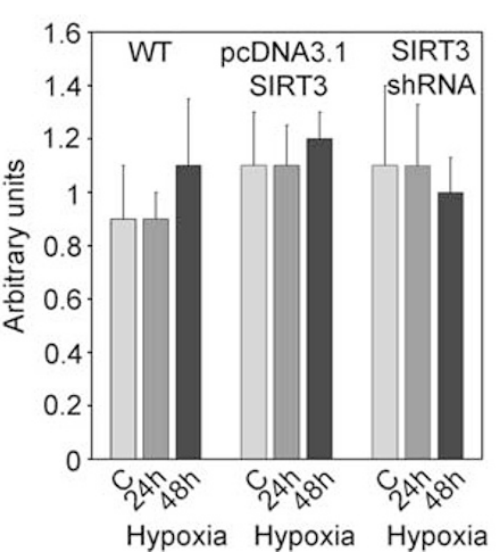

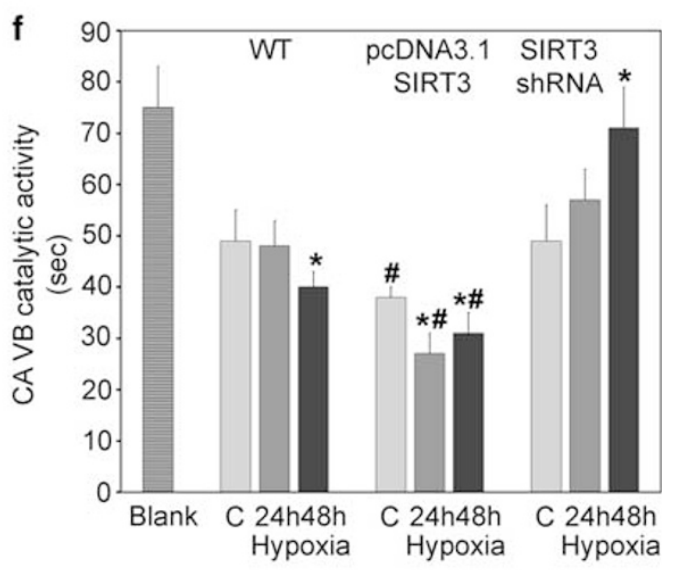


hypoxia (Figure 6c). In order to demonstrate the necessary role of SP1 sites, three constructs were obtained in which luciferase activity is under the control of SIRT3 promoter. In particular, construct A contains all SP1 sites, construct B has none of SP1 sites and construct $E$ is missing three SP1 sites. Figure 6d shows that SP1 sites are important to have a SIRT3 promoter activity and that all SP1 sites are required to have an efficient promoter activity. In fact, we obtained an upregulation in construct $A$ when all SP1 sites were present and a similar downregulation in constructs $B$ and $E$ irrespectively if all or only three SP1 sites were missing (Figure 6d).

\section{Discussion}

In the present study, we demonstrated that increased expression of SIRT3 protected cells from hypoxia or STSinduced cell death by inhibiting induction of MPT, loss of membrane potential and ROS accumulation. In particular, we showed that SIRT3 activated CA VB catalytic activity thereby maintaining a physiological $\mathrm{pH}_{\mathrm{i}}$, and inhibited HKII mitochondrial binding thereby decreasing the probability of PTP opening. These two events reduced Bax mitochondrial accumulation and activation of the mitochondrial apoptotic pathway.

In our system we documented that SIRT3 has an important role in protecting hypoxic cells from both apoptosis and necrosis. It is known that tumor cells undergo glycolysis even in the presence of ample oxygen. ${ }^{28} \mathrm{HKII}$ has a high affinity for glucose and is highly expressed in most tumors localizing on the OMM. ${ }^{29}$ Recently, SIRT3 has been shown to induce HKII detachment from OMM by deacetylating cyclophilin D, thereby increasing oxidative phosphorylation. ${ }^{12}$ Our results demonstrate that under hypoxia SIRT3 overexpression causes a detachment of HKII from the mitochondria with a decrease in glycolytic rate and a reduction of lactate. Importantly, SIRT3 silencing increased both HKII binding to the mitochondrial fraction and lactate production. HKII increased expression and switch to glycolysis by the tumor cells, accumulates intracellular protons that under 'normal' conditions are decreased by oxidative phosphorylation. ${ }^{30}$ Moreover, for the first time, we also show that SIRT3 can regulate $\mathrm{pH}_{\mathrm{i}}$ by controlling the activity of the mitochondrial enzyme CA VB. Very little is known about CA VB, however, this enzyme is localized in the mitochondrial matrix and it is ubiquitously expressed. ${ }^{25}$ Recently, CA VB has been shown to convert the $\mathrm{CO}_{2}$ produced by TCA cycle and $\beta$-oxidation to $\mathrm{HCO}_{3}^{-}$which, in turn controls metabolic pathways increasing oxidative phosphorylation and reducing ROS generation. ${ }^{31}$ Moreover, we speculate that the increased CA VB catalytic activity in SIRT3-overexpressing cells, by increasing $\mathrm{HCO}_{3}^{-}$ production, can buffer protons formed when ATP from cytosolic glycolysis is hydrolyzed by the F1F0 ATPase. ${ }^{32}$ The net result of SIRT3 effects on HKII and CA VB is a tight control of $\mathrm{pH}_{\mathrm{i}}$. In fact, a drop in $\mathrm{pH}_{\mathrm{i}}$ can activate the proapoptotic protein Bax. ${ }^{33}$ Once activated, Bax translocates in the mitochondria inducing the MPT. ${ }^{34}$ This sequence of events is reproduced in our model where intracellular acidification followed by mitochondrial accumulation of Bax is more evident in hypoxic SIRT3-silenced cells. By contrast, SIRT3 overexpression prevented intracellular acidification, Bax activation and MPT induction. In fact, only in SIRT3silenced cells we observed an increase in cytochrome $c$ release, PARP cleavage, nuclear translocation of AIF and cell death. Previously, protection from apoptotic cell death due to an SIRT3-increased expression has been described in cardiomyocytes where Ku70, a DNA-repair factor and inhibitor of Bax-mediated apoptosis, is deacetylated by SIRT3 hindering the translocation of Bax to mitochondria. ${ }^{35}$ Herewith, we propose an alternative or parallel mechanism for SIRT3 protection. SIRT3 by increasing CA VB catalytic activity and by inhibiting HKII mitochondrial binding reduces the glycolytic rate and decreases intracellular acidification. This latter event prevents Bax activation and mitochondrial translocation as well as induction of the MPT.

We also observed that SIRT3 protected cells from necrosis. Our results show that overexpression of SIRT3 reduced LDH as well as HMGB1 release. In particular, HMGB1 is considered a reliable measure of necrosis because it is released only by necrotic cells whereas remains associated to DNA in apoptotic cells. ${ }^{17}$ When SIRT3 was silenced ROS, LDH and HMGB1 accumulation were increased both under normoxia and hypoxia.

Figure 4 SIRT3 regulates HKII mitochondrial binding and CA catalytic activity. (a) MDA-MB-231 WT, SIRT3-overexpressing and SIRT3-silenced cells were incubated under normoxia or hypoxia for 36 and $72 \mathrm{~h}$. Whole extracts or mitochondrial and cytosolic fractions were obtained. HKII content was measured as described under Materials and Methods. Data are representative of at least three separate experiments. Phb was used as loading control for mitochondria, whereas $\beta$-actin was used as a loading control for cytosol. Numbers below each blot represent average HKIl level relative to either Phb or $\beta$-actin. *Significantly different from control normoxic cells (C). " Significantly different from control normoxic WT and SIRT3-overexpressing cells. Significance was set at $P<0.05$. (b) MDA-MB-231 WT, SIRT3-overexpressing and SIRT3-silenced cells were incubated under normoxia or hypoxia for $48 \mathrm{~h}$. Lactate accumulation in culture medium was measured as described under Materials and Methods. *Significantly different from normoxia and hypoxia $48 \mathrm{~h}$ of WT and SIRT3-silenced cells. "Significantly different from normoxia and hypoxia of WT and SIRT3-overexpressing cells. Significance was set at $P<0.05$. C: control normoxic cells. (c) MDA-MB-231 WT, SIRT3-overexpressing and SIRT3-silenced cells were incubated under normoxia or hypoxia for 24 and $48 \mathrm{~h}$. Whole extracts were obtained and CA IX content measured as described under Materials and Methods. Data are representative of at least three separate experiments. $\beta$-actin was used as a loading control for total lysate. Histograms on the right side represent average CA IX level relative to $\beta$-actin. *Significantly different from C. ${ }^{\#}$ Significantly different from control normoxic WT and SIRT3-overexpressing cells. Significance was set at $P<0.05$. (d) MDA-MB-231 WT, SIRT3-overexpressing and SIRT3-silenced cells were incubated under normoxia or hypoxia for 24 and $48 \mathrm{~h}$. CA IX catalytic activity, in seconds, was measured in intact cells as described under Materials and Methods. Data are representative of at least three separate experiments. Blank represents chemical reaction without cells. *Significantly different from C. "Significantly different from control normoxic WT and SIRT3-overexpressing cells. Significance was set at $P<0.05$. (e) MDA-MB-231 WT, SIRT3-overexpressing and SIRT3-silenced cells were incubated under normoxia or hypoxia for 24 and $48 \mathrm{~h}$. Mitochondrial fractions were obtained and CA VB content measured as described under Materials and Methods. Data are representative of at least three separate experiments. Phb was used as a loading control for mitochondrial lysates. Histograms on the right side represent average CA VB level relative to Phb. (f) MDA-MB-231 WT, SIRT3-overexpressing and SIRT3-silenced cells were incubated under normoxia or hypoxia for 24 and $48 \mathrm{~h}$. CA VB catalytic activity, in seconds, was measured in mitochondrial extracts as described under Materials and Methods. Data are representative of at least three separate experiments. Blank represents chemical reaction without cells. *Significantly different from $\mathrm{C}$. "Significantly different from normoxic and hypoxic WT and SIRT3-silenced cells. Significance was set at $P<0.05$ 

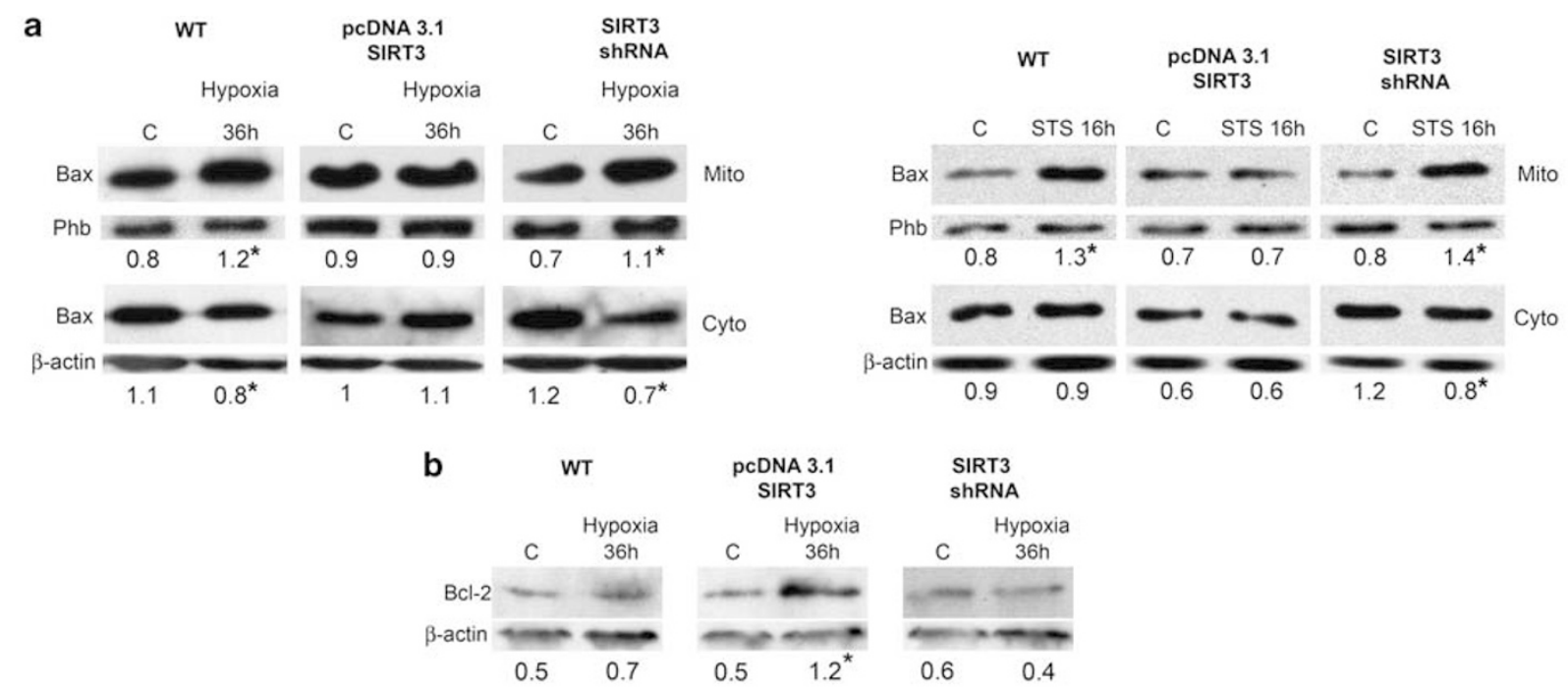

C
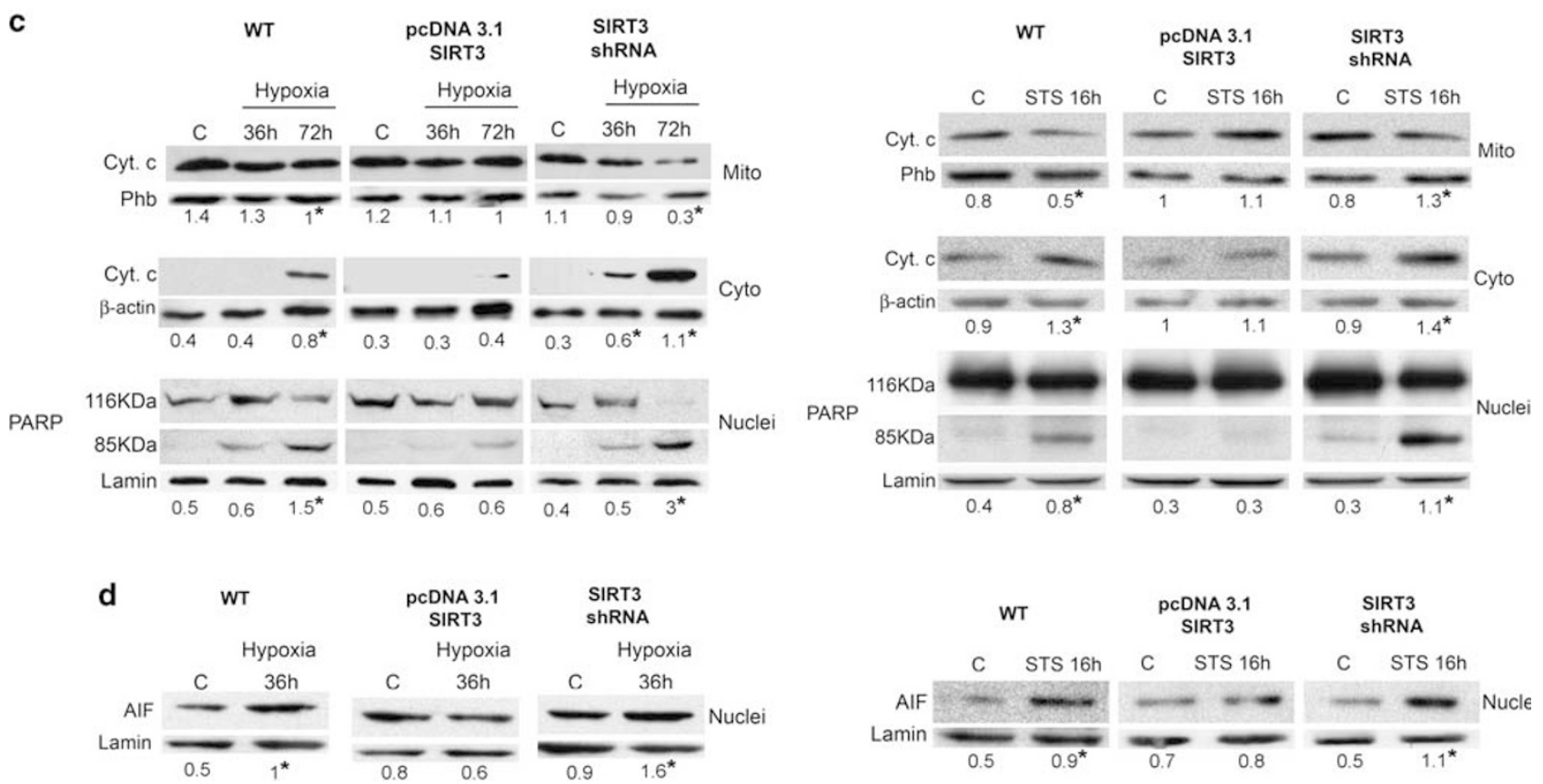

Figure 5 SIRT3 effects on mitochondrial apoptotic pathway. (a) MDA-MB-231 WT, SIRT3-overexpressing and SIRT3-silenced cells were incubated under normoxia or hypoxia for $36 \mathrm{~h}$. Cells were then processed to obtain mitochondrial and cytosolic fractions. Bax levels were assessed by western blot on mitochondria and cytosol as described under Materials and Methods. Data are representative of at least three separate experiments. Phb was used as loading control for mitochondria whereas $\beta$-actin was used as a loading control for cytosol. Numbers below each blot represent average level of Bax protein, relative to either Phb or $\beta$-actin. *Significantly different from control normoxic cells (C). Significance was set at $P<0.05$. (b) MDA-MB-231 WT, SIRT3-overexpressing and SIRT3-silenced cells were incubated under normoxia or hypoxia for $36 \mathrm{~h}$. Cells were then processed to obtain whole-cell lysates. Bcl-2 levels were assessed by western blot as described under Materials and Methods. Data are representative of at least three separate experiments. $\beta$-Actin was used as a loading control. Numbers below each blot represent average level of Bcl-2 protein, relative to $\beta$-actin. ${ }^{*}$ Significantly different from $\mathrm{C}$. Significance was set at $P<0.05$. (c) MDA-MB-231 WT, SIRT3-overexpressing and SIRT3-silenced cells were incubated under normoxia, hypoxia or STS for the times indicated. Cells were then processed and nuclear, mitochondrial and cytosolic fractions obtained. Cytochrome $c$ levels were assessed by western blot on mitochondria and cytosol as described under Materials and Methods. PARP cleavage was, instead, measured on nuclei as described under Materials and Methods. Data are representative of at least three separate experiments. Phb was used as loading control for mitochondria whereas $\beta$-actin was used as a loading control for cytosol. Lamin was used as nuclear loading control. Numbers below each blot represent average level of the indicated protein, relative to either Phb or $\beta$-actin or lamin. *Significantly different from C. Significance was set at $P<0.05$. (d) MDA-MB-231 WT, SIRT3-overexpressing and SIRT3-silenced cells were incubated under normoxia, hypoxia or STS for the times indicated. Cells were then processed and nuclear fractions obtained. Nuclear translocation of AIF was measured on nuclei as described under Materials and Methods. Data are representative of at least three separate experiments. Lamin was used as loading control for nuclei. Numbers below each blot represent average AIF level relative to lamin. ${ }^{*}$ Significantly different from $\mathrm{C}$. Significance was set at $P<0.05$

Interestingly, we also showed that SIRT3 expression increased under hypoxia as part of an adaptive survival mechanism to such metabolic stress that depends by the transcription factor SP1.
On the basis of our results we have drawn a schematic illustration to explain the central role of SIRT3 in mitochondrial cell death (Figure 7). SIRT3 is involved in the reduction of ROS, an event that to one side diminishes LDH and HMGB1 
a

b
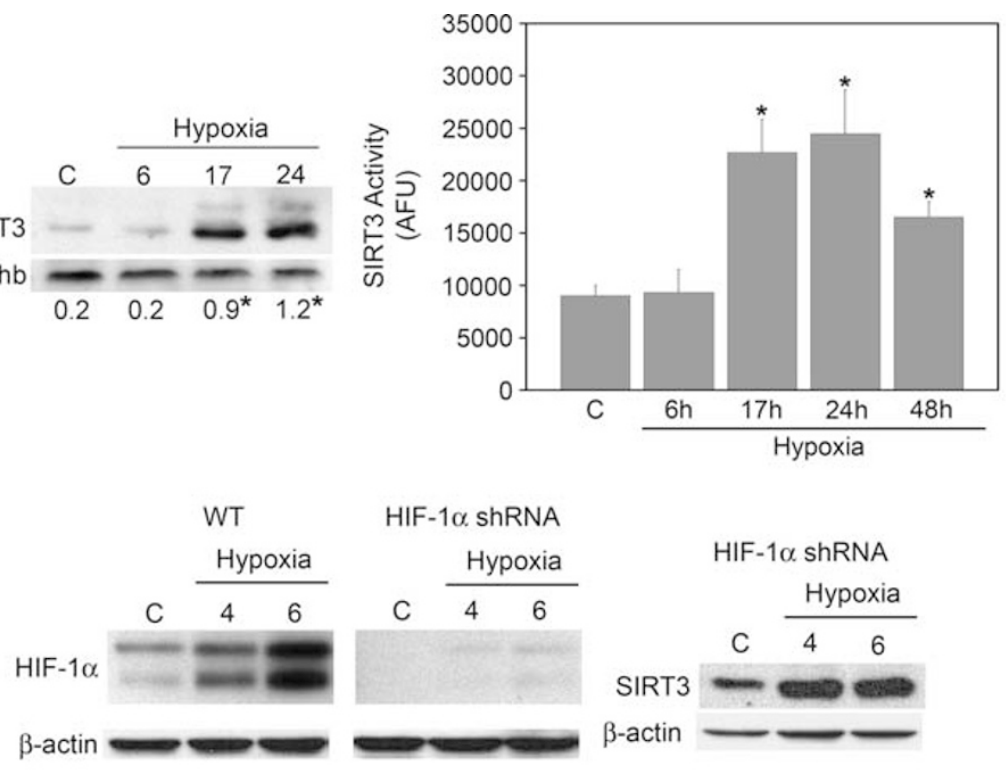

C

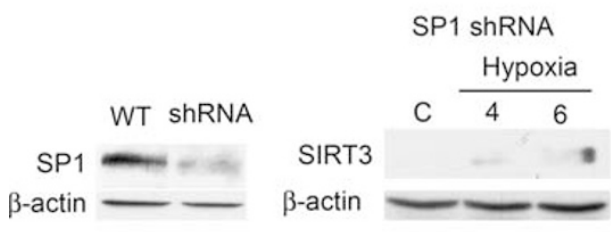

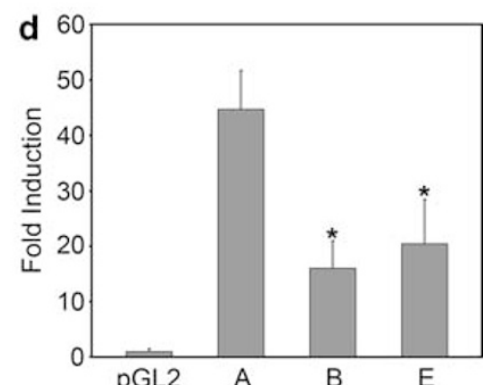

Figure 6 SP1 regulates SIRT3 increase under hypoxia. (a) Left side, MDA-MB-231 cells were incubated under normoxic or hypoxic conditions. After the times indicated, cells were processed to obtain a mitochondrial extracts as described under Materials and Methods. The contents of SIRT3 were determined by western blotting. Phb was used as loading control for the mitochondrial fraction. Blots are representative of at least three separate experiments. Numbers below the blots represent average SIRT3 level, relative to Phb. *Significantly different from control normoxic cells $(C)$. Significance was set at $P<0.05$. Right side, MDA-MB-231 cells were incubated under normoxic or hypoxic conditions. After the times indicated, SIRT3 deacetylase activity was measured on mitochondrial fractions as described under Materials and Methods. Data are representative of at least three separate experiments. Values are represented as mean \pm S.D. *Significantly different from control normoxic cells. Significance was set at $P<0.05$. (b) MDA-MB-231 cells either silenced for HIF-1 $\alpha$ or transduced with lentiviral empty control particles were incubated under normoxic or hypoxic conditions. After the times indicated, cells were processed to obtain a whole-cell extracts as described under Materials and Methods. The content of HIF-1 $\alpha$ (left side) and SIRT3 (right side) were determined by western blotting. $\beta$-Actin was used as a whole cells loading control. Blots are representative of at least three separate experiments. C, control normoxic cells. (c) MDA-MB-231 either silenced for SP1 or transduced with lentiviral empty control particles were processed to obtain a whole-cell extract as described under Materials and Methods. The content of SP1 was determined by western blotting (left side). SP1-silenced cells were incubated under normoxic or hypoxic conditions for the times indicated. The content of SIRT3 was measured by western blotting (right side). $\beta$-Actin was used as a loading control. Blots are representative of at least three separate experiments. C, control normoxic cells. (d) Luciferase expression after transient transfection of pGL2 plasmid containing multiple SP1 binding sites of SIRT3 promoter (A), pGL2 plasmid deleted of all the SP1 binding sites of SIRT3 promoter (B) and pGL2 plasmid deleted of three SP1 binding sites of SIRT3 promoter (E) with respect to the pGL2-basic vector. The values reported are the means \pm S.D. of three independent experiments. ${ }^{*}$ Significantly different from plasmid A. Significance was set at $P<0.05$

release reducing necrosis, and to the other prevents MPT induction reducing apoptosis. At the same time SIRT3 induces HKII detachment from mitochondrial membrane reducing glycolitic rate and increases catalytic activity of mitochondrial CA VB thereby controlling $\mathrm{pH}_{\mathrm{i}}$. The molecular mechanism through which SIRT3 controls CA VB activity is presently under study. We presume that SIRT3 could deacetylate CA VB, thereby increasing its activity. In fact, SIRT3 and CA VB are both in the mitochondrial matrix and CA VB presents predicted acetylation sites (lysines) as assessed by the online predictor for protein acetylation PAIL, (prediction of acetylation on internal lysines). ${ }^{36}$ In conclusion, by controlling intracellular acidification, SIRT3 reduces Bax activation and mitochondrial translocation inhibiting mitochondrial apoptosis.

\section{Materials and Methods}

Cell cultures. The MDA-MB-231 human breast carcinoma cell line, the human epithelial carcinoma cell line HeLa and human chronic myelogenous leukemia cell line K562 were purchased from LGC Standards (Milan, Italy). MDA-MB-231, HeLa and K562 were maintained in 75-cm2 polystyrene flasks (Corning Costar Corp., Oneonta, NY, USA) with RPMI 1640 medium (Mediatech, Inc., Herndon, VA, USA), containing 100 units $/ \mathrm{ml}$ penicillin, $0.1 \mathrm{mg} / \mathrm{ml}$ streptomycin, and $10 \%$ heatinactivated fetal bovine serum. Cells were maintained at $37^{\circ} \mathrm{C}$ in a humidified atmosphere of $5 \% \mathrm{CO}_{2}$ and $95 \%$ air.

Treatment protocols and antibodies. Hypoxia was achieved by incubating cells in a hypoxic chamber (Billups-Rothenberg Inc., Del Mar, CA, USA) where a $1 \%$ oxygen mix was flushed in for 4 min according to the manufacturer's instructions.

Staurosporine (Sigma-Aldrich, St Louis, MO, USA) was dissolved in dimethyl sulfoxide (DMSO) as $1 \mathrm{mM}$ stock solution and added to a final concentration of $250 \mathrm{nM}$. 


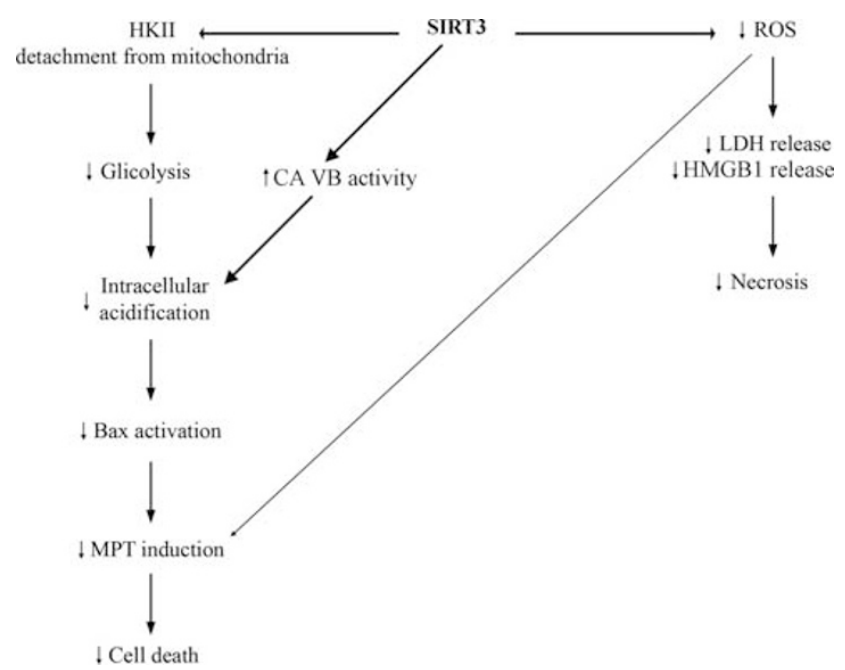

Figure 7 Schematic representation of the pathways controlled by SIRT3. SIRT3 increases cellular resistance to both necrosis and apoptosis by regulating HKII binding to the mitochondria, CA VB catalytic activity and ROS production

Cyclosporin A (Biomol Research Laboratories, Plymouth Meeting, PA, USA) was dissolved in DMSO and added at a final concentration of $5 \mu \mathrm{M}$.

NAC (Sigma-Aldrich) was dissolved in RPMl and added at a final concentration of $5 \mathrm{mM}$.

The following primary antibodies were used: rabbit anti-SIRT3 antibody, rabbit anti-AIF (Cell Signaling Technology, Inc., Danvers, MA, USA), mouse anti-HIF-1 $\alpha$, mouse anti-lamin (BD Bioscience, San Jose, CA, USA), mouse anticytochrome $c$, mouse antiprohibitin, rabbit anti-SP1, rabbit anti-CA IX and mouse anti-CA VB (Novus Biologicals, Littleton, CO, USA), mouse anti- $\beta$ actin, rabbit anti-Bax (N-20), mouse anti-Bcl-2, goat anti-HKII, mouse anti-PARP (Santa Cruz Biotechnology, Santa Cruz, CA, USA) and rabbit anti-HMGB1 (Sigma-Aldrich).

The following secondary antibodies were used: mouse anti-rabbit HRP, goat antimouse HRP (Amersham Biosciences, Piscataway, NJ, USA) and donkey anti-goat HRP (Santa Cruz Biotechnology).

Generation of stable SIRT3 transfectants. MDA-MB-231 cells were stably transfected with a pcDNA3.1 expression vector encoding for human SIRT3Flag (Addgene Inc., Cambridge, MA, USA). Stable clones were generated by delivering plasmid DNA constructs into cells using TurboFectin 8.0 (Origene Technologies, Rockville, MD, USA) according to the manufacturer's recommendations. Briefly, cells were seeded on a 24-well plate. The following day the cells were transfected. Turbofectin reagents were first mixed with serum-free RPMI at room temperature for $5 \mathrm{~min}$. Subsequently, plasmid DNA was added to the TurboFectin-containing media and incubated at room temperature for $30 \mathrm{~min}$. After that the mixtures were added to the cells. The selection of stable clones was started $24 \mathrm{~h}$ later with the addition of $500 \mu \mathrm{g} / \mathrm{ml}$ of Geneticin (Sigma-Aldrich).

Lentiviral transduction. Mission TRC short hairpin RNA (shRNA) lentiviral transduction particles expressing shRNA targeting SIRT3, SP1, HIF- $1 \alpha$ and lentiviral negative control particles were purchased from Sigma-Aldrich. Stably transduced clones were generated according to the manufacturer's instructions. Briefly, cells were seeded on a 24-well plate. The following day cells were infected. After $24 \mathrm{~h}$ medium was changed with fresh RPMI. Selection of stable clones was started $24 \mathrm{~h}$ later with the addition of $3 \mu \mathrm{g} / \mathrm{ml}$ of puromycin. The expression of SIRT3, SP1, HIF-1 $\alpha$ was confirmed by western blotting.

Annexin V/PI staining. Cells $\left(2 \times 10^{6}\right)$ were plated in $100 \mathrm{~mm}$ dishes. Following treatment the cells were washed twice in PBS, collected and centrifuged at 2000 r.p.m. for $10 \mathrm{~min}$ at $4{ }^{\circ} \mathrm{C}$. Percentage of apoptotic and necrotic cells was quantified with the FITC Annexin V apoptosis detection kit I (BD Bioscience) according to manufacturer's recommendations. Briefly, cell pellets were resuspended in $1 \mathrm{ml}$ of $1 \mathrm{X}$ binding buffer and $100 \mu \mathrm{l}$ of the suspension were transferred in a $5 \mathrm{ml}$ tube. FITC Annexin V $(5 \mu \mathrm{l})$ and $\mathrm{PI}(5 \mu \mathrm{l})$ were added to the cells for $15 \mathrm{~min}$ at room temperature. Afterward, $400 \mu \mathrm{l}$ of $1 \mathrm{X}$ binding buffer were added to each tube and analyzed with a FACSCalibur (BD Biosciences) flow cytometer.
Hoechst staining. Cells were seeded on glass coverslips in a 6-well plate. Following treatment nuclei were stained by addition of Hoechst (Sigma-Aldrich) staining solution at a final concentration of $62.5 \mathrm{ng} / \mathrm{ml}$. After $15 \mathrm{~min}$ incubation cells were washed twice and fixed in $3.7 \%$ paraformaldehyde for $10 \mathrm{~min}$. After two washes in PBS, coverslips were mounted and stained nuclei observed under fluorescence microscope (Carl Zeiss, Milan, Italy).

Isolation of nuclear, cytosolic and mitochondrial fractions. Cells $\left(2 \times 10^{6}\right)$ were plated in $100 \mathrm{~mm}$ dishes. Following treatment the cells were scraped off the plate using a rubber policeman and centrifuged at 2000 r.p.m. for $10 \mathrm{~min}$ at $4^{\circ} \mathrm{C}$. The cell pellets were resuspended in $1 \mathrm{ml}$ of $20 \mathrm{mM} \mathrm{HEPES}-\mathrm{KOH}$, pH 7.5, $10 \mathrm{mM} \mathrm{KCl}, 1.5 \mathrm{mM} \mathrm{MgCl}$, $1 \mathrm{mM}$ EDTA, $1 \mathrm{mM}$ EGTA, $1 \mathrm{mM}$ phenylmethylsulfonyl fluoride, $10 \mu \mathrm{g} / \mathrm{ml}$ leupeptin, $10 \mu \mathrm{g} / \mathrm{ml}$ aprotinin and $250 \mathrm{mM}$ sucrose. The cells were broken open with six passages through a 26-gauge needle applied to a $1 \mathrm{ml}$ syringe. The homogenate was centrifuged at $1000 \mathrm{~g}$ for $5 \mathrm{~min}$ at $4{ }^{\circ} \mathrm{C}$ to harvest nuclei. The supernatant was transferred to a high-speed centrifuge tube and centrifuged at $10000 \mathrm{~g}$ for $25 \mathrm{~min}$ at $4^{\circ} \mathrm{C}$. The resulting supernatant (cytosolic fraction) was concentrated through a Microcon YM-10 Centrifugal Filter Device (Millipore, Bedford, MA, USA), whereas the pellet (mitochondrial fraction) was lysed in $50 \mathrm{mM}$ Tris (tris(hydroxymethyl)aminomethane)-HCl, $150 \mathrm{mM} \mathrm{NaCl}, 1 \%$ Triton X-100, $0.1 \%$ Nonidet P-40, $1 \mathrm{mM}$ phenylmethylsulfonyl fluoride, $10 \mu \mathrm{g} / \mathrm{ml}$ leupeptin, $10 \mu \mathrm{g} / \mathrm{ml}$ aprotinin). Protein concentration of the fraction was determined by the Bradford assay (Bio-Rad, Hercules, CA, USA). For all experiments, the purity as well as equal loading of nuclear fraction was determined by measuring levels of nuclear protein lamin. The purity as well as equal loading of mitochondrial fraction was determined by measuring levels of mitochondrial protein prohibitin (Phb). The purity as well as equal loading of cytosolic fraction was determined by measuring levels of cytosolic protein $\beta$-actin.

Protein extraction and western blot assay. Cells $\left(1 \times 10^{6}\right)$ for wholecell lysate were pelleted at $700 \times g\left(5 \mathrm{~min}\right.$ at $\left.4{ }^{\circ} \mathrm{C}\right)$ and lysed in $50 \mu$ l of cell lysis buffer (20 mM Tris, pH 7.4, $100 \mathrm{mM} \mathrm{NaCl}, 1 \%$ Triton, $1 \mathrm{mM}$ phenylmethylsulfonyl fluoride, $10 \mu \mathrm{g} / \mathrm{ml}$ leupeptin, $10 \mu \mathrm{g} / \mathrm{ml}$ aprotinin). After $30 \mathrm{~min}$ on ice, the lysates were clarified by centrifugation $\left(10 \mathrm{~min}\right.$ at $\left.4^{\circ} \mathrm{C}\right)$ and the supernatant collected. Protein concentration was determined by the Bradford assay (Bio-Rad). Equivalent amounts of protein were electrophoresed on SDS-polyacrylamide gels. Kaleidoscope prestained standards (Bio-Rad) were used to determine molecular weight. The gels were then electroblotted onto PVDF membranes. After blocking with 5\% milk, membranes were incubated with the primary antibody overnight. Finally, the relevant protein was visualized by staining with the appropriate secondary horseradish peroxidase-labeled antibody for $1 \mathrm{~h}$ followed by enhanced chemiluminescence.

Measurement of the $\Delta \Psi_{\mathrm{mt}}$. The change in $\Delta \Psi_{\mathrm{mt}}$, was analyzed by flow cytometry using the $\Delta \Psi_{\mathrm{mt}}$ sensitive dye 5,5',6,6'-tetrachloro-1,1',3,3'-tetraethylbenzimidazolylcarbocyanine iodide (JC-1). Cells $\left(1 \times 10^{4}\right.$ for normoxic condition and $6 \times 10^{4}$ for hypoxic condition) were cultured in 24-well plate. Following treatment cells were harvested by centrifugation at 1000 r.p.m. for $5 \mathrm{~min}$ at room temperature. The cell pellets were resuspended in $0.5 \mathrm{ml}$ of cell culture media containing $10 \mu \mathrm{m} \mathrm{JC}-1$, incubated for $15 \mathrm{~min}$ at $37^{\circ} \mathrm{C}$ in $5 \% \mathrm{CO}_{2}$ and immediately transferred on ice. Cell staining was determined by FACSCalibur (BD Biosciences). Two light-scattering parameters, the forward scatter (cell size) and side scatter (intracellular granularity and complexity), and two fluorescence parameters, fluorescence channel 1 (FL1) and fluorescence channel 2 (FL2), were measured.

Measurement of intracellular ROS. To determine the amount of ROS production, $2^{\prime}, 7^{\prime}$-dichlorfluorescein-diacetate (DCFH-DA) was used. Following treatment cells were harvested by centrifugation at 1200 r.p.m. for $5 \mathrm{~min}$ at room temperature. The pellets were resuspended in $0.5 \mathrm{ml}$ of cell culture media containing $50 \mu \mathrm{M}$ DCFH-DA and incubated for $30 \mathrm{~min}$ at $37^{\circ} \mathrm{C}$ in $5 \% \mathrm{CO}_{2}$. Fluorescence intensity was measured by flow cytometry (FACScalibur, BD Biosciences).

CA activity measurements. CA catalytic activity was determined using a slightly modified end-point titration method of Maren. ${ }^{37}$ Briefly, intact cell (for CA IX activity) or mitochondrial extracts (for CA VB activity) were resuspended in $500 \mu \mathrm{l}$ of ice-cold assay buffer ( $20 \mathrm{mM}$ imidazole, $5 \mathrm{mM}$ Tris, $0.2 \mathrm{mM}$-nitrophenol). The cuvette containing the sample and assay buffer was placed in a Lambda 35 UV/VIS (Perkin Elmer Instruments, Waltham, MA, USA) spectrophotometer and $500 \mu \mathrm{l}$ of ice-cold $\mathrm{CO}_{2}$-saturated $\mathrm{H}_{2} \mathrm{O}$ was added into the cuvette. The exact time in seconds for the yellow color disappearance was counted. In the control experiments without cells or extracts, the color disappeared in about $75 \mathrm{sec}$. 
Intracellular and extracellular $\mathrm{pH}$ determination. Cytosolic $\mathrm{pH}$ was evaluated by flow cytometry using the pH-sensitive fluorescent probe BCECF-AM (Sigma-Aldrich). Following treatment cells were harvested by centrifugation and the pellets were resuspended in culture medium containing $20 \mu \mathrm{mol} / \mathrm{I} \mathrm{BCECF-AM}$ (2,7-bis(2-carboxyethyl)-5-(and-6)-carboxyfluorescein acetoxymethyl ester) and incubated for $30 \mathrm{~min}$ at $37^{\circ} \mathrm{C}$ in $5 \% \mathrm{CO}_{2}$. The cells were then washed in $\mathrm{HBSS}$, placed on ice, and analyzed with a FACSCalibur equipped with a $488 \mathrm{~nm}$ argon laser collecting the emission of BCECF-AM in the FL1 and FL2 channels. To obtain a calibration curve, about $10^{7}$ cells were kept at $37^{\circ} \mathrm{C}$ for $30 \mathrm{~min}$ in $1 \mathrm{ml} \mathrm{RPMl} \mathrm{containing}$ $20 \mu \mathrm{mol} / \mathrm{I}$ BCECF-AM and incubated with different potassium phosphate buffers in a range of $\mathrm{pH}$ from 5.5 to $7.5 \mathrm{in}$ the presence of nigericin $(10 \mu \mathrm{mol} / \mathrm{l})$ (Sigma-Aldrich).

Extracellular $\mathrm{pH}$ was determined in the culture medium with a pH211 pHmeter (Hanna Instruments, Milan, Italy).

Analysis of lactate production and LDH activity. Accumulation of lactate in the culture medium was determined using lactate assay kit (Biovision, Mountain View, CA, USA) according to the manufacturer's protocol.

The activity of LDH was analyzed using the LDH Cytotoxicity Assay Kit II (BioVision), and the assay was performed as described below. Following treatment culture media were collected after centrifugation at $600 \mathrm{~g}$ for $10 \mathrm{~min}$ The clear medium was transferred into an optically clear 96 -well plate containing the reaction mixture and incubated for $30 \mathrm{~min}$ at room temperature. Following incubation, the absorbance of solution was measured at $450 \mathrm{~nm}$ by using a microplate reader. The assays were performed in triplicate and the results were compared with the control values according to the manufacturer's instructions.

SIRT3 deacetylase activity assay. SIRT3 deacetylase activity was determined using a SIRT3 Fluorimetric Activity Assay/Drug Discovery Kit (Biomol Research Laboratories) following the manufacturer's protocol. In brief, the mitochondrial extract $(5 \mu \mathrm{g})$ was incubated with the Fluor de Lys substrate buffer at $37^{\circ} \mathrm{C}$ for $1 \mathrm{~h}$ followed by incubation with fluor de lys developer at $37^{\circ} \mathrm{C}$ for $40 \mathrm{~min}$. After excitation at $360 \mathrm{~nm}$, emitted light was detected at $460 \mathrm{~nm}$ using Infinte 200 microplate fluorometer (Tecan, Männedorf, Switzerland). The fluorescence intensity of the assay buffer was subtracted from each experimental sample.

SIRT3 promoter construction and luciferase assay. Construction of reporter gene plasmids, deletion analysis as well as luciferase assay were performed as previously described. ${ }^{27}$

Statistical analysis. The results are expressed as means \pm S.D. and $95 \%$ confidence intervals $(95 \% \mathrm{Cl})$. Before using parametric tests, the assumption of normality was verified using the Shapiro-Wilk $W$-test. Student's paired $t$-test was used to determine any significant differences before and after treatment. Significance was set at $0.05(P \leq 0.05)$. SPSS statistical software package (SPSS Inc., Version 13.0.1 for Windows Chicago, IL, USA) was used for all statistical calculations.

\section{Conflict of Interest}

The authors declare no conflict of interest.

Acknowledgements. This work was supported by the Italian Ministry of Health (contribution institutional research funding 2011).

1. Dutnall RN, Pillus L. Deciphering NAD-dependent deacetylases. Cell 2001; 105: 161-164.

2. Guarente L, Kenyon $\mathrm{C}$. Genetic pathways that regulate ageing in model organisms. Nature 2000; 408: 255-262.

3. Haigis MC, Guarente LP. Mammalian sirtuins-emerging roles in physiology, aging, and calorie restriction. Genes Dev 2006; 20: 2913-2921.

4. Michan S, Sinclair D. Sirtuins in mammals: insights into their biological function. Biochem J 2007; 404: 1-13.

5. Saunders LR, Verdin E. Sirtuins: critical regulators at the crossroads between cancer and aging. Oncogene 2007; 26: 5489-5504.

6. Brachmann CB, Sherman JM, Devine SE, Cameron EE, Pillus L, Boeke JD. The SIR2 gene family, conserved from bacteria to humans, functions in silencing, cell cycle progression, and chromosome stability. Genes Dev 1995; 9: 2888-2902.
7. Sauve AA, Celic I, Avalos J, Deng H, Boeke JD, Schramm VL. Chemistry of gene silencing: the mechanism of NAD +-dependent deacetylation reactions. Biochemistry 2001; 40: 15456-15463.

8. Imai S, Armstrong CM, Kaeberlein M, Guarente L. Transcriptional silencing and longevity protein Sir2 is an NAD-dependent histone deacetylase. Nature 2000; 403: 795-800.

9. Michishita E, Park JY, Burneskis JM, Barrett JC, Horikawa I. Evolutionarily conserved and nonconserved cellular localizations and functions of human SIRT proteins. Mol Biol Cell 2005; 16: 4623-4635.

10. Verdin E, Hirschey MD, Finley LW, Haigis MC. Sirtuin regulation of mitochondria: energy production, apoptosis, and signaling. Trends Biochem Sci 2010; 35: 669-675.

11. Lombard DB, Alt FW, Cheng HL, Bunkenborg J, Streeper RS, Mostoslavsky R et al. Mammalian Sir2 homolog SIRT3 regulates global mitochondrial lysine acetylation. Mol Cell Biol 2007; 27: 8807-8814.

12. Shulga N, Wilson-Smith R, Pastorino JG. Sirtuin-3 deacetylation of cyclophilin D induces dissociation of hexokinase II from the mitochondria. J Cell Sci 2010; 123: 894-902.

13. Giorgio V, Soriano ME, Basso E, Bisetto E, Lippe G. Forte Ma et al. Cyclophilin D in mitochondrial pathophysiology. Biochim Biophys Acta 2010; 1797: 1113-1118.

14. Shulga N, Pastorino JG. Ethanol sensitizes mitochondria to the permeability transition by inhibiting deacetylation of cyclophilin-D mediated by sirtuin-3. J Cell Sci 2010; 123: 4117-4127.

15. Li Y, Johnson N, Capano M, Edwards M, Crompton M. Cyclophilin-D promotes the mitochondrial permeability transition but has opposite effects on apoptosis and necrosis. Biochem J 2004; 383: 101-109

16. Schubert A, Grimm S. Cyclophilin D a component of the permeability transition-pore, is an apoptosis repressor. Cancer Res 2004; 64: 85-93.

17. Krysko DV, Vanden Berghe T, Parthoens E, D'Herde K, Vandenabeele P. Methods for distinguishing apoptotic from necrotic cells and measuring their clearance. Methods Enzymol 2008; 442: 307-341.

18. Susin SA, Zamzami N, Kroemer G. Mitochondria as regulators of apoptosis: doubt no more. Biochim Biophys Acta 1998; 1366: 151-165.

19. Liu X, Kim CN, Yang J, Jemmerson R, Wang X. Induction of apoptotic program in cell-free extracts: requirement for dATP and cytochrome c. Cell 1996; 86: 147-157.

20. Nechushtan A, Smith CL, Hsu YT, Youle RJ. Conformation of the Bax C-terminus regulates subcellular location and cell death. EMBO J 1999; 18: 330-341.

21. Tafani M, Cohn JA, Karpinich NO, Rothman RJ, Russo MA, Farber JL. Regulation of intracellular pH mediates Bax activation in HeLa cells treated with staurosporine or tumor necrosis factor-alpha. J Biol Chem 2002; 277: 49569-49576.

22. Mathupala SP, Ko YH, Pedersen PL. Hexokinase II cancer's double-edged sword acting as both facilitator and gatekeeper of malignancy when bound to mitochondria. Oncogene 2006; 25: 4777-4786

23. Qiu X, Brown K, Hirschey MD, Verdin E, Chen D. Calorie restriction reduces oxidative stress by SIRT3-mediated SOD2 activation. Cell Metab 2010; 12: 662-667.

24. Madshus IH. Regulation of intracellular pH in eukaryotic cells. Biochem J 1988; 250: 1-8.

25. Gilmour KM. Perspectives on carbonic anhydrase. Comp Biochem Physiol A Mol Integr Physiol 2010; 157: 193-197.

26. Penninger JM, Kroemer G. Mitochondria, AIF and caspases-rivaling for cell death execution. Nat Cell Biol 2003; 5: 97-99.

27. Bellizzi D, Dato S, Cavalcante P, Covello G, Di Cianni F, Passarino G et al. Characterization of a bidirectional promoter shared between two human genes related to aging: SIRT3 and PSMD13. Genomics 2007; 89: 143-150.

28. Warburg O. On the origin of cancer cells. Science 1956; 123: 309-314.

29. Mathupala SP, Rempel A, Pedersen PL. Glucose catabolism in cancer cells: identification and characterization of a marked activation response of the type II hexokinase gene to hypoxic conditions. J Biol Chem 2001; 276: 43407-43412.

30. Vághy PL. Role of mitochondrial oxidative phosphorylation in the maintenance of intracellular pH. J Mol Cell Cardiol 1979; 11: 933-940.

31. Acin-Perez R, Salazar E, Kamenetsky M, Buck J, Levin LR, Manfredi G. Cyclic AMP produced inside mitochondria regulates oxidative phosphorylation. Cell Metab 2009; 9 : 265-276.

32. Solaini G, Baracca A, Lenaz G, Sgarbi G. Hypoxia and mitochondrial oxidative metabolism. Biochim Biophys Acta 2010; 1797: 1171-1177.

33. Cartron PF, Oliver L, Mayat E, Meflah K, Vallette FM. Impact of pH on Bax alpha conformation, oligomerisation and mitochondrial integration. FEBS Lett 2004; 578: 41-46.

34. Green DR, Reed JC. Mitochondria and apoptosis. Science 1998; 28: 1309-1312.

35. Sundaresan NR, Samant SA, Pillai VB, Rajamohan SB, Gupta MP. SIRT3 is a stressresponsive deacetylase in cardiomyocytes that protects cells from stress-mediated cell death by deacetylation of Ku70. Mol Cell Biol 2008; 28: 6384-6401.

36. Li A, Xue Y, Jin C, Wang M, Yao X. Prediction of Nepsilon-acetylation on internal lysines implemented in Bayesian Discriminant Method. Biochem Biophys ResCommun 2006; 1 : 818-824.

37. Maren TH. A simplified micromethod for the determination of carbonic anhydrase and its inhibitors. J Pharmacol Exp Ther 1960; 130: 26-29. 